\title{
ON SOME DIFFERENTIAL GEOMETRIC CHARACTERIZA- TIONS OF A BUNDLE-LIKE METRIC
}

\author{
By Haruo Kitahara and Shinsuke Yorozu
}

\section{§1. Introduction.}

We have known some interesting theorems about the behaviour of geodesics of a bundle-like metric:

Theorem A (Y. Muto [6]). A geodesic of a fibred riemannian manifold tangent to an "allowed curve" at one point is always an "allowed curve" if and only if "the fibres are parallel".

Theorem B (B. L. Reinhart [10]). A geodesıc of a bundle-like metric is orthogonal at one point if and only if it is orthogonal at every point.

The topological obstructions for the existence of the foliation with bundlelike metric were studied by R. Sacksteder [11], J. S. Pasternack $[8,9]$ and others. But of the conditions for the given riemannian metric of a foliated manifold to be a boundle-like metric very little is definitely known (cf. [5]). Of cource, not all foliations have bundle-like metrics [10]. The completeness of a bundle-like metric was studied by one of the authors $[3,4]$.

In this note, we will give some differential geometric conditions for a given riemannian metric $\langle$,$\rangle on a foliated riemannian manifold of codimension one to$ be a bundle-like metric in terms of geodesics. Our main theorem is the following:

THEOREM C. Suppose that a foliated riemannian manifold is of codimension one and that all leaves are totally geodesic with respect to the given riemannian metric $\langle$,$\rangle . Then the metric \langle$,$\rangle is a bundle-like metric with respect to the$ foliation if and only if all geodesics with "angle $\alpha$ " to a leaf at one point have "constant angle $\alpha$ " to each leaf at every point.

Furthermore, we can give a proof of the following theorem:

THEOREM D. The given riemannian metric $\langle$,$\rangle on a foliated riemannian$ manifold of codimension one is a bundle-like metric with respect to the foliation if and only if all geodesics orthogonal to a leaf at one point are orthogonal to each leaf at every point.

Received October 13, 1977. 
We remark that this theorem was announced by A. M. Naveira [7]. theorems.

And we will give some typical examples in connection with the above

\section{$\S 2$. Notations.}

We shall be in $C^{\infty}$-category. Let $M$ be a connected $(p+q)$-dimensional riemannian manifold with riemannian metric $\langle$,$\rangle and riemannian connection \nabla$ with respect to $\langle$,$\rangle . A foliated riemannian manifold M$ of codimension $q$ has, by definition, an integrable subbundle $E$ of fibre dimension $p$ of the tangent bundle $T M$ over $M$. Then $M$ is covered by the flat coordinate charts $\left\{\left(U, x^{1}, \cdots, x^{p}\right.\right.$, $\left.\left.x^{p+1}, \cdots, x^{p+q}\right)\right\}$ (cf. [10]). Let $Q$ be the quotient bundle $T M / E$. The restriction of a vector bundle to $U$ and the set of all cross-sections of a vector bundle are denoted by $\left.\cdot\right|_{U}$ and $\Gamma(\cdot)$ respectively. The natural projection $\pi: T M \rightarrow Q$ induces a map $\pi: \Gamma(T M) \rightarrow \Gamma(Q)$. $[$,$] denotes the bracket operator. Adopted the$ following ranges of indices and the corresponding summation convention; $1 \leqq A$, $B, C, \cdots \leqq p+q, 1 \leqq \imath, \jmath, k, \cdots \leqq p$ and $p+1 \leqq \alpha, \beta, \gamma, \cdots \leqq p+q$.

\section{$\S 3$. Bott partial connection and bundle-like metric.}

DEFINITION 3.1. In each flat coordinate chart $\left(U ; x^{A}\right)$, a frame $\left\{X_{1}, \cdots, X_{p}\right.$, $\left.X_{p+1}, \cdots, X_{p+q}\right\}$ is an adapted frame to $E$ if $\left\{X_{1}, \cdots, X_{p}\right\}$ and $\left\{\pi\left(X_{p+1}\right), \cdots\right.$, $\left.\pi\left(X_{p+q}\right)\right\}$ span $\Gamma\left(\left.E\right|_{U}\right)$ and $\Gamma\left(\left.Q\right|_{U}\right)$ respectively.

In each $\left(U ; x^{A}\right)$, frames $\left\{\partial / \partial x^{2}, \partial / \partial x^{\alpha}\right\}$ and $\left\{\partial / \partial x^{2}, \partial / \partial x^{\alpha}-t_{\alpha}{ }^{k} \partial / \partial x^{k}\right\}\left(t_{\alpha}{ }^{k}\right.$ are functions on $U$ ) are adapted frames to $E$ (cf. [8,9], [10], [12, 13]).

DEFINITION 3.2. In each flat coordinate chart $\left(U ; x^{A}\right)$, an adapted frame $\left\{E_{\imath}, E_{\alpha}\right\}$ to $E$ is called a basıc adapted frame to $E$ if $E_{\imath}=\partial / \partial x^{\imath}$ and $E_{\alpha}=\partial / \partial x^{\alpha}$ $-t_{\alpha}{ }^{k} \partial / \partial x^{k}$.

For a basic adapted frame $\left\{E_{\imath}, E_{\alpha}\right\}$,

$$
\pi\left(\left[X, E_{\alpha}\right]\right)=0 \quad \text { for any } \quad X \in \Gamma\left(\left.E\right|_{U}\right)
$$

holds.

In each $\left(U ; x^{A}\right)$, by the suitable choice of functions $t_{\alpha}{ }^{k}$, the given riemannian metric $\langle$,$\rangle has a local expression$

$$
\left.\langle,\rangle\right|_{U}=g_{\imath j}\left(x^{k}, x^{\ddot{r}}\right) \theta^{2} \otimes \theta^{j}+g_{\alpha \beta}\left(x^{k}, x^{r}\right) \theta^{\alpha} \otimes \theta^{3},
$$

where $\left\{\theta^{\imath}, \theta^{\alpha}\right\}$ is the dual frame of the adapted frame $\left\{E_{\imath}, E_{\alpha}\right\}$ (cf. [10], [12]). We notice that $\operatorname{det}\left(g_{\imath \jmath}\right) \neq 0$ implies $\operatorname{det}\left(g_{\alpha_{\beta}}\right) \neq 0$. Then the metric $\langle$,$\rangle induces a$ metric «, 》 on $Q$, that is, for any $S_{1}, S_{2} \in \Gamma\left(\left.Q\right|_{U}\right)$,

$$
\ll S_{1},\left.S_{2} 》\right|_{U}=g_{\alpha \beta}\left(x^{k}, x \ddot{i}\right) \theta^{\alpha}\left(\widetilde{S}_{1}\right) \theta^{s}\left(\widetilde{S}_{2}\right),
$$

where $\pi\left(\widetilde{S}_{1}\right)=S_{1}$ and $\pi\left(\widetilde{S}_{2}\right)=S_{2}$ (cf. [8]). $\ll, \gg$ is well-defined. 
Definition 3.3. A map $\hat{\nabla}: \Gamma(E) \times \Gamma(Q) \rightarrow \Gamma(Q)$ is defined by

$$
\hat{\nabla}_{X} S=\pi([X, \tilde{S}])
$$

for any $X \in \Gamma(E)$ and $S \in \Gamma(Q)$, where $\tilde{S} \in \Gamma(T M)$ such that $\pi(\tilde{S})=S$. The map $\hat{\nabla}$ is well-defined by the integrability of $E$. $\hat{\nabla}$ is called the Bott partıal connection on $Q$ (cf. [1]).

Definition 3.4. The Bott partial connection $\hat{\nabla}$ on $Q$ is metrical with respect to $\ll, \gg$ if

$$
\ll \hat{\nabla}_{X} S_{1}, S_{2} \gg+\ll S_{1}, \hat{\nabla}_{X} S_{2} \gg=X \ll S_{1}, S_{2} 》
$$

for any $X \in \Gamma\left(\left.E\right|_{U}\right)$ and any $S_{1}, S_{2} \in \Gamma\left(\left.Q\right|_{U}\right)$.

DEFINITION 3.5. The riemannian metric $\langle$,$\rangle is a bundlelike metric with$ respect to $E$ (or with respect to the foliation) if, in its local expression (3.2),

$$
\partial g_{\alpha \beta} / \partial x^{\imath}=0,
$$

that is, $g_{\alpha_{\beta} \beta}\left(x^{k}, x^{r}\right)=g_{\alpha \beta}\left(x^{r}\right)$.

Take the basic adapted frame $\left\{E_{i}, E_{\alpha}\right\}$ to $E$ in each $\left(U, x^{A}\right)$. We notice that (3.6) means

$$
E_{\imath}\left\langle E_{\alpha}, E_{\beta}\right\rangle=0 \text {. }
$$

LEMMA 3.1. If the riemannian metric $\langle$,$\rangle is a bundlelike metric with respect$ to $E$, then the Bott partial connection $\hat{\nabla}$ is metrical with respect to the metric $\ll$, 》.

Proof. Take the basic adapted frame $\left\{E_{\imath}, E_{\alpha}\right\}$ to $E$. For any $S_{1}, S_{2} \in \Gamma\left(\left.Q\right|_{U}\right)$, suppose that $\widetilde{S}_{i}=f_{1}^{i} E_{\imath}+f_{1}^{\alpha} E_{\alpha}$ and $\widetilde{S}_{2}=f_{2}^{i} E_{\imath}+f_{2}^{\alpha} E_{\alpha}$ satisfy $\pi\left(\widetilde{S}_{1}\right)=S_{1}$ and $\pi\left(\widetilde{S}_{2}\right)=S_{2}$ respectively. Then, from (3.3), (3.4) and (3.6), we have

and

$$
\begin{aligned}
\ll \hat{\nabla}_{E_{j}} S_{1}, S_{2} 》 & =\ll \pi\left(\left[E_{\jmath}, \tilde{S}_{1}\right]\right), S_{2} \gg \\
& =g_{\alpha \beta}\left(x^{\gamma}\right) \theta^{\alpha}\left(\left[E_{\jmath}, \widetilde{S}_{1}\right]\right) \theta^{3}\left(\tilde{S}_{2}\right) \\
& =g_{\alpha_{\beta} \beta}\left(x^{\check{r}}\right) E_{j}\left(f_{1}^{\alpha}\right) f_{2}^{\beta},
\end{aligned}
$$

$$
\begin{aligned}
\ll \hat{\nabla}_{E_{j}} & S_{1}, S_{2} \gg+\ll S_{1}, \hat{\nabla}_{E_{j}} S_{2} 》 \\
& =g_{\alpha_{\beta} \beta}\left(x^{r}\right) E_{j}\left(f_{1}^{\alpha}\right) f_{2}^{\beta}+g_{\alpha_{\beta} \beta}\left(x^{r}\right) f_{1}^{\alpha} E_{j}\left(f_{2}^{\beta}\right) \\
& =E_{j}\left(g_{\alpha_{\beta}}\left(x^{\check{r}}\right) f_{1}^{\alpha} f_{2}^{\beta}\right) \\
& =E_{j}\left(g_{\alpha_{\beta} \beta}\left(x^{\check{r}}\right) \theta^{\alpha}\left(\tilde{S}_{1}\right) \theta^{\beta}\left(\tilde{S}_{2}\right)\right) \\
& =E_{j} \ll S_{1}, S_{2} 》 .
\end{aligned}
$$

Therefore, we have 


$$
\ll \hat{\nabla}_{X} S_{1}, S_{2} \gg+\ll S_{1}, \hat{\nabla}_{X} S_{2} 》=X \ll S_{1}, S_{2} 》
$$

for any $X \in \Gamma\left(\left.E\right|_{U}\right)$.

q.e.d.

\section{$\S 4$. Proofs of THEOREM C, D and examples.}

The following lemma is our fundamental tool.

LEMMA 4.1. The metric $\langle$,$\rangle is a bundle-like metruc if and only if, for each$ flat coordinate chart $\left(U ; x^{A}\right)$, there exists an orthonormal adapted frame $\left\{X_{\imath}, X_{\alpha}\right\}$ to $E$ such that $\hat{\nabla}_{X} \pi\left(X_{\alpha}\right)=0$ for any $X \in \Gamma\left(\left.E\right|_{U}\right)$.

Proof. Suppose that the metric $\langle$,$\rangle is a bundle-like metric. We have an$ orthonormal frame $\left\{X_{\imath}, X_{\alpha}\right\}$ from the basic adapted frame $\left\{E_{\imath}, E_{\alpha}\right\}$ to $E$ by the Schmidt's orthogonalization process to $\left\{E_{\imath}\right\}$ and $\left\{E_{\alpha}\right\}$ respectively. Clearly, $\left\{X_{1}, \cdots, X_{p}\right\}$ and $\left\{\pi\left(X_{p+1}\right), \cdots, \pi\left(X_{p+q}\right)\right\}$ span $\Gamma\left(\left.E\right|_{U}\right)$ and $\Gamma\left(\left.Q\right|_{U}\right)$ respectively. Then $\left\{X_{\imath}, X_{\alpha}\right\}$ is an orthonormal adapted frame to $E$. By (3.1), $\left\langle E_{\alpha}, E_{\beta}\right\rangle=g_{\alpha_{\beta}}\left(X^{\tau}\right)$ implies $\pi\left(\left[X_{\imath}, X_{\alpha}\right]\right)=0$. Therefore, we have $\hat{\nabla}_{X} \pi\left(X_{\alpha}\right)=0$ for any $X \in \Gamma\left(\left.E\right|_{U}\right)$.

Conversely, let $\left\{X_{\imath}, X_{\alpha}\right\}$ be an orthonormal adapted frame to $E$ such that $\hat{\nabla}_{X} \pi\left(X_{\alpha}\right)=0$ for any $X \in \Gamma\left(\left.E\right|_{U}\right)$. Since the basic adapted frame $\left\{E_{\imath}, E_{\alpha}\right\}$ to $E$ satisfies $\left\langle E_{\imath}, E_{\alpha}\right\rangle=0$, we can write $E_{\alpha}$ of form $E_{\alpha}=h_{\alpha}^{\beta} X_{\beta}$ where $h_{\alpha}^{\beta}$ are functions on $U$. Then we have

$$
\begin{aligned}
\hat{\nabla}_{E_{\imath}} \pi\left(E_{\alpha}\right) & =\pi\left(\left[E_{\imath}, E_{\alpha}\right]\right) \\
& =\pi\left(\left[E_{\imath}, h_{\alpha}^{\beta} X_{\beta}\right]\right) \\
& =h_{\alpha}^{\beta} \pi\left(\left[E_{\imath}, X_{\beta}\right]\right)+E_{i}\left(h_{\alpha}^{\beta}\right) \pi\left(X_{i}\right) \\
& =h_{\alpha}^{\beta} \hat{\nabla}_{E_{i}} \pi\left(X_{\beta}\right)+E_{i}\left(h_{\alpha}^{\beta}\right) \pi\left(X_{, 3}\right) \\
& =E_{i}\left(h_{\alpha}^{\beta}\right) \pi\left(X_{\beta}\right) .
\end{aligned}
$$

And, by (3.1), $\hat{\nabla}_{E_{i}} \pi\left(E_{\alpha}\right)=\pi\left(\left[E_{\imath}, E_{\alpha}\right]\right)=0$. Then we have $E_{i}\left(h_{\alpha}^{\beta}\right) \pi\left(X_{\beta}\right)=0$, and $E_{\imath}\left(h_{\alpha}^{\beta}\right)$ $=0$ by the linearly independence of $\pi\left(X_{\beta}\right)$. Thus we have

$$
\begin{aligned}
E_{\imath}\left\langle E_{\alpha}, E_{\beta}\right\rangle & =E_{i}\left\langle h_{\alpha}^{\tau} X_{\gamma}, h_{\beta}^{\tau} X_{-}\right\rangle \\
& =E_{\imath}\left(h_{\alpha}^{\gamma} h_{\beta}^{\tau} \delta_{\gamma \tau}\right) \\
& =0 .
\end{aligned}
$$

Therefore, by (3.7), the metric $\langle$, $\rangle$ is a bundle-like metric.

q. e.d.

THEOREM 4.1. The metric $\langle$,$\rangle is a bundle-like metric if and only if, for$ each flat coordinate chart $\left(U ; x^{A}\right)$, there exists an orthonormal adapted frame $\left\{X_{\imath}, X_{\alpha}\right\}$ to $E$ such that $\left\langle\nabla_{X_{\alpha}} X_{\imath}, X_{\beta}\right\rangle+\left\langle\nabla_{X_{\beta}} X_{\imath}, X_{\alpha}\right\rangle=0$.

Proof. Suppose that the metric $\langle$, $\rangle$ is a bundle-like metric. By lemma 3.1, 
there exists an orthonormal adapted frame $\left\{X_{2}, X_{\alpha}\right\}$ to $E$ such that $\hat{\nabla}_{X} \pi\left(X_{\alpha}\right)=0$ for any $X \in \Gamma\left(\left.E\right|_{U}\right)$. Then we have

$$
\begin{aligned}
\left\langle\nabla_{X_{\alpha}} X_{\imath}, X_{\beta}\right\rangle & =\left\langle\nabla_{X_{i}} X_{\alpha}, X_{\beta}\right\rangle-\left\langle\left[X_{\imath}, X_{\alpha}\right], X_{\beta}\right\rangle \\
& =\left\langle\nabla_{X_{i}} X_{\alpha}, X_{\beta}\right\rangle,
\end{aligned}
$$

since $\hat{\nabla}_{X_{i}} \pi\left(X_{c}\right)=0$ implies $\left[X_{\imath}, X_{\alpha}\right] \in \Gamma\left(\left.E\right|_{U}\right)$. Therefore, we have

$$
\begin{aligned}
\left\langle\nabla_{X_{\alpha}} X_{\imath}\right. & \left., X_{\beta}\right\rangle+\left\langle\nabla_{X_{\beta}} X_{\imath}, X_{\alpha}\right\rangle \\
& =\left\langle\nabla_{X_{i}} X_{\alpha}, X_{\beta}\right\rangle+\left\langle\nabla_{X_{i}} X_{\beta}, X_{\alpha}\right\rangle \\
& =X_{i}\left\langle X_{\alpha}, X_{\beta}\right\rangle \\
& =0 .
\end{aligned}
$$

Conversely, suppose that there exists an orthonormal adapted frame $\left\{X_{\imath}, X_{\alpha}\right\}$ to $E$ such that $\left\langle\nabla_{X_{\alpha}} X_{\imath}, X_{\beta}\right\rangle+\left\langle\nabla_{X_{\beta}} X_{\imath}, X_{\alpha}\right\rangle=0$. Then we can write the basic adapted frame $\left\{E_{\imath}, E_{\alpha}\right\}$ to $E$ of form $E_{i}=h_{\imath}^{\jmath} X$, and $E_{\alpha}=h_{\alpha}^{\gamma} X_{\gamma}\left(h_{i}^{3}\right.$ and $h_{\alpha}^{\gamma}$ are functions on $U$ ). Thus we have

$$
\begin{aligned}
E_{i}\left\langle E_{\alpha}, E_{\beta}\right\rangle & \\
= & \left\langle\nabla_{E_{i}} E_{\alpha}, E_{\beta}\right\rangle+ \\
= & \left\langle h_{i}^{\jmath} h_{\beta}^{\tau}\left(X_{j}, \nabla_{E_{i}} E_{\beta}^{\gamma}\right) \delta_{i \tau}+h_{\alpha}^{\gamma}\left\langle\left[X_{\jmath}, X_{i}\right], X_{\tau}\right\rangle\right) \\
& +h_{i}^{j} h_{\alpha}^{\gamma} h_{\beta}^{\tau}\left\langle\nabla_{X_{\tau}} X_{\jmath}, X_{\tau}\right\rangle \\
& +h_{i}^{j} h_{\alpha}^{\gamma}\left(X_{j}\left(h_{\beta}^{\tau}\right) \delta_{i \tau}+h_{\beta}^{\tau}\left\langle X_{i},\left[X_{\jmath}, X_{\tau}\right]\right\rangle\right) \\
& +h_{i}^{j} h_{\alpha}^{\gamma} h_{\beta}^{\tau}\left\langle X_{\gamma}, \nabla_{X_{\tau}} X_{\jmath}\right\rangle .
\end{aligned}
$$

Since we have

$$
\begin{gathered}
X_{\jmath}\left(h_{c}^{\gamma}\right) \delta_{\gamma \tau}+h_{\alpha}^{\gamma}\left\langle\left[X_{\jmath}, X_{\gamma}\right], X_{\tau}\right\rangle \\
=X_{\jmath}\left(h_{\alpha}^{\gamma}\right) \delta_{\gamma_{\tau}}+\left\langle\left[X_{\jmath}, h_{\alpha}^{\gamma} X_{\gamma}\right], X_{\vec{\tau}}\right\rangle \\
\quad-\left\langle X_{\jmath}\left(h_{\alpha}^{\gamma}\right) X_{\gamma}, X_{\tau}\right\rangle \\
=\left\langle\left[X_{\jmath}, E_{\alpha}\right], X_{\vec{\tau}}\right\rangle \\
=0 \quad \text { (from }(3.1)),
\end{gathered}
$$

we have

$$
\begin{aligned}
E_{i}\left\langle E_{\alpha},\right. & \left.E_{\beta}\right\rangle \\
& =h_{i}^{j} h_{\alpha}^{\gamma} h_{\beta}^{\tau}\left(\left\langle\nabla_{X_{\tau}} X_{\jmath}, X_{\tau}\right\rangle+\left\langle\nabla_{X_{\tau}} X_{\jmath}, X_{\gamma}\right\rangle\right) \\
& =0 .
\end{aligned}
$$

Therefore, the metric $\langle$,$\rangle is a bundle-like metric.$

q.e.d. 
For each flat coordinate chart $\left(U ; x^{A}\right)$, let $\left\{w^{2}, w^{\alpha}\right\}$ be the dual frame of an orthonormal adapted frame $\left\{X_{\imath}, X_{\alpha}\right\}$ to $E$, that is, $w^{A}\left(X_{B}\right)=\delta_{B}^{A}$. Let $\sigma(t)$ and $Z(t)$ be a curve in $U$ and a vector field along $\sigma(t)$ respectively. Then we have

$$
\begin{aligned}
w^{A}\left(\nabla_{\dot{\sigma}(t)} Z(t)\right)= & \frac{d}{d t}\left(w^{A}(Z(t))\right) \\
& +w^{B}(Z(t)) w_{B}^{A}(\dot{\sigma}(t)),
\end{aligned}
$$

where $\dot{\sigma}(t)=d \sigma(t) / d t$ and $d w^{A}=w^{B} \wedge w_{B}^{A}$ (cf. [14] p. 19 (1.6.12)). If we put $w_{B}^{A}$ $=\Gamma_{C B}^{A} w^{C}$, then we have $\nabla_{X_{C}} X_{B}=\Gamma_{C B}^{A} X_{A}$ and

$$
\Gamma_{C B}^{A}+\Gamma_{C A}^{B}=0 \text {. }
$$

(4.1) implies

$$
\begin{aligned}
w^{A}\left(\nabla_{\dot{\sigma}(t)} Z(t)\right)= & \frac{d}{d t}\left(w^{A}(Z(t))\right) \\
& +\Gamma_{C B}^{A} w^{B}(Z(t)) w^{C}(\dot{\sigma}(t)) .
\end{aligned}
$$

Proof of THEOREM D.

Let $\sigma(t)$ be any geodesic orthogonal to each leaf at every point parametrized by arc-length. By (4.3) we have

$$
\begin{aligned}
w^{\imath}\left(\nabla_{\dot{\sigma}(t)} \dot{\sigma}(t)\right)= & \frac{d}{d t}\left(w^{\imath}(\dot{\sigma}(t))\right) \\
& +\Gamma_{j k}^{i} w^{k}(\dot{\sigma}(t)) w^{j}(\dot{\sigma}(t)) \\
& +\Gamma_{j p+1}^{i} w^{p+1}(\dot{\sigma}(t)) w^{j}(\dot{\sigma}(t)) \\
& +\Gamma_{p+1 j}^{i} w^{j}(\dot{\sigma}(t)) w^{p+1}(\dot{\sigma}(t)) \\
& +\Gamma_{p+1}^{i}{ }_{p+1} w^{p+1}(\dot{\sigma}(t)) w^{p+1}(\dot{\sigma}(t)) .
\end{aligned}
$$

But $\nabla_{\dot{\sigma}(t)} \dot{\sigma}(t)=0$ and $w^{\imath}(\dot{\sigma}(t))=0$ imply

$$
\Gamma_{p+1}^{i} p+1 w^{p+1}(\dot{\sigma}(t)) w^{p+1}(\dot{\sigma}(t))=0 .
$$

Then we have $\Gamma_{p+1}^{i} p_{p+1}=0$, and this equality with (4.2) implies

$$
\Gamma_{p+1}^{p+1}=0 \text {. }
$$

Then we have

$$
\left\langle\nabla_{X_{p+1}} X_{\imath}, X_{p+1}\right\rangle=0
$$

Therefore, by theorem 4.1, the metric $\langle$, $\rangle$ is a bundle-like metric.

The converse assertion is clear from theorem $B$. q.e.d.

Definition 4.1. Suppose that $M$ is a foliated riemannian manifold of codimension one (if necessarily, $M$ is supposed to be transversally orientable). A geodesic $\sigma(t)$ parametrized by arc-length has an angle $\alpha\left(t_{0}\right)$ to a leaf at $t=t_{0}$ if, 
in a flat coordinate chart $\left(U ; x^{A}\right)$ containing the point $\sigma\left(t_{0}\right),\left.\left\langle\dot{\sigma}(t), X_{p+1}\right\rangle\right|_{t=t_{0}}$ $=\cos \alpha\left(t_{0}\right) \neq 0$ holds for an orthonormal adapted frame $\left\{X_{1}, \cdots, X_{p}, X_{p+1}\right\}$ to $E$. $\sigma(t)$ has the constant angle $\alpha$ to each leaf at every point if $\alpha(t)=$ constant $=\alpha$, that is, $\left\langle\dot{\sigma}(t), X_{p+1}\right\rangle=$ constant $=\cos \alpha \neq 0$.

Proof of THEOREM $C$.

We have, from (4.3),

(4.2) implies

$$
\begin{aligned}
& w^{p+1}\left(\nabla_{\sigma(t)} \dot{\sigma}(t)\right) \\
&=\frac{d}{d t}\left(w^{p+1}(\dot{\sigma}(t))\right) \\
& \quad+\Gamma_{i j}^{p+1} w^{j}(\dot{\sigma}(t)) w^{2}(\dot{\sigma}(t)) \\
& \quad+\Gamma_{p+1 i}^{p+1} w^{\imath}(\dot{\sigma}(t)) w^{p+1}(\dot{\sigma}(t)) \\
&+\Gamma_{\imath+1}^{p+1} w^{p+1}(\dot{\sigma}(t)) w^{\imath}(\dot{\sigma}(t)) \\
&+\Gamma_{p+1}^{p+1} p+1 \\
&
\end{aligned}
$$

$$
\Gamma_{\imath+1}^{p+1}=\Gamma_{p+1}^{p+1} p+1=0
$$

Since all leaves are totally geodesic, we have

$$
\Gamma_{\imath j}^{p+1}=0 .
$$

Suppose that a geodesic $\sigma(t)$ satisfies $\left\langle\dot{\sigma}(t), X_{p+1}\right\rangle=$ constant $\neq 0$. Then we have

$$
\begin{gathered}
\frac{d}{d t}\left(w^{p+1}(\dot{\sigma}(t))\right)=0 \text { and } \\
\nabla_{\dot{\sigma}(t)} \dot{\sigma}(t)=0 .
\end{gathered}
$$

Then, from (4.4), we have

$$
\Gamma_{p+1}^{p+1}{ }_{i} w^{2}(\dot{\sigma}(t)) w^{p+1}(\dot{\sigma}(t))=0,
$$

and

$$
\Gamma_{p+1}^{p \perp 1} w^{2}(\dot{\sigma}(t))=0 \quad(\text { by }(4.7)) .
$$

As, by assumpsion, $w^{2}(\dot{\sigma}(t))$ are arbitrary,

$$
\Gamma_{p+1}^{p+1}=0,
$$

that is, $\left\langle\nabla_{X_{p+1}} X_{2}, X_{p+1}\right\rangle=0$. Therefore, the metric $\langle$,$\rangle is a bundle-like metric.$

Conversely, let a geodesic $\sigma(t)$ parametrized by arc-length have an angle $\alpha$ to a leaf at $t=t_{0}$. It is sufficient to prove that $\left\langle\dot{\sigma}(t), X_{p+1}\right\rangle=\operatorname{constan} t=\cos \alpha$ for an orthonormal adapted frame $\left\{X_{1}, \cdots, X_{p}, X_{p+1}\right\}$ to $E$ on $\left(U ; x^{A}\right)$ containing the point $\sigma\left(t_{0}\right)$. (4.4), (4.5), (4.6) and (4.8) imply 


$$
\frac{d}{d t}\left(u^{p+1}(\dot{\sigma}(t))\right)=0
$$

that is, $\left\langle\dot{\sigma}(l), X_{p+1},=\right.$ constant $=\cos \alpha$. Therefore, $\sigma(t)$ has the constant angle $\alpha$ to each leaf at every point.

q. e. d.

EXAMPLE 4.1. A family of irrational spirals on a flat torus $T^{2}$ of dimension two defines a foliation with a bundle-like metric whose leaves are totally geodesic. Then any geodesic has the constant angle $\alpha$ to each leaf at every point.

EXAMPLE 4.2. Let $R^{2}$ be a two dimensional euclidean space and $M=R^{2}$ $-\{(0,0)\}$. Then $M$ is a foliated manifold whose leaves are $L_{r}=\left\{(x, y) \subseteq M \mid x^{2}+y^{2}\right.$ $\left.=r^{2}, r>0\right\}$. The canonical metric of $R^{2}$ induces a bundle-like metric on $M$. Then each leaf $L_{r}$ is not totally geodesic, and, for example, a geodesic $y=c(c$ is a non-zero constant) has distinct angle to each leaf at every point.

EXAMPLE 4.3. Let $R^{3}$ be a three dimensional euclidean space and

$$
S^{2}=\left\{(x, y, z) \in R^{3} \mid x^{2}+v^{2}+z^{2}=1\right\}
$$

with canonical metric $<$, . If we put $x=-\cos \varphi \sin \theta, y=\sin \varphi \sin \theta$ and $z=\cos \theta$, then $S^{2}=\{(\varphi, \theta) \mid 0 \leqq \varphi<2 \pi, 0 \leq \theta<\pi\}$. For any $\varepsilon>0$, we set $S_{\varepsilon}^{\prime}=\left\{(\varphi, \theta) \in S^{2} \mid \varepsilon<\theta\right.$ $<\pi-\varepsilon\}$. A family of the great circular parts given by $\varphi=$ constant defines a foliation on $S_{\S}^{2}$ whose leaves are totally geodesic with respect to the restricted metric $\left.\langle\rangle\right|_{,s_{\varepsilon}^{2}}$ of $\langle$,$\rangle on S_{\varepsilon}^{2}$. But the metric $\left.\zeta,\right\rangle\left.\right|_{s_{\varepsilon}^{2}}$ is not a bundlelike metric with respect to the foliation. Let $\sigma(t)$ be a great circle (geodesic) on $S_{\bar{s}}^{\text {? }}$ through the two points $\left(\varphi_{0}, \theta_{0}\right)$ and $\left(\varphi_{1}, \theta_{0}\right)$ where $\varphi_{0}+\pi \equiv \varphi_{1}(\bmod 2 \pi)$ and $\theta_{0}$ $\neq \pi / 2$. Then $\sigma(t)$ is not orthogonal to the leaf defined by $\varphi=\varphi_{0}$, but orthogonal to the leaf defined by $\varphi=\left(\varphi_{0}+\varphi_{1}\right) / 2$.

Remark. In the case of codimension $q \geqq 2$, suppose that the bundle $Q$ (or the orthogonal complement $E^{-}$of $E$ ) is trivial. Let $\left\{X_{\imath}, X_{a}\right\}$ be an orthonormal adapted frame to $E$. And suppose that a geodesic $\sigma(t)$ parametrized by arclength has the same normal cosme $l_{a t}(t)$ at $t=t_{0}$, that is,

$$
l_{p+1}\left(t_{0}\right)=l_{p+2}\left(t_{0}\right)=\cdots=l_{p+q}\left(t_{0}\right) \neq 0 .
$$

where the normal cosine $l_{\pi}(t)$ is defined by

$$
\left\langle\dot{\sigma}(t), X_{r}\right\rangle=l_{\alpha}(t) .
$$

If the metric $\langle$,$\rangle is a bundle-like metric and all leaves are totally geodesic,$ then, for each $t$,

$$
l_{p+1}(t)=l_{p+2}(t)=\cdots=l_{p+q}(t)=\text { constant }=l_{p+1}\left(t_{0}\right) .
$$

EXAMPLE 4.4. Let $R^{n}$ be an $n$-dimensional euclidean space and $S^{n-1}(r)$ an $(n-1)$-dimensional standard sphere of radius $r$ in $R^{n} . \quad \boldsymbol{Q}$ and $\boldsymbol{Q}^{*}$ are the quaternion number field and the pure quaternion respectively. We can identify $\left(x^{1}, x^{2}, x^{3}, x^{4}\right) \in R^{4}$ with $x^{1} \cdot 1+x^{2} \cdot \imath+x^{3} \cdot y+x^{4} \cdot k \in \boldsymbol{Q}$ and $\left(y^{1}, y^{2}, y^{3}\right) \in R^{3}$ with $y^{1} \cdot i+y^{2} \cdot y+y^{3} \cdot k € \boldsymbol{Q}^{*}$. Then we write $S^{3}(1)$ and $S^{2}(1 / 2)$ of forms $\{a \in \boldsymbol{Q}\|a\|=1\}$ 
and $\left\{b \in \boldsymbol{Q}^{*} \mid\|b\|=1 / 2\right\}$ respectively, where $\|\cdot\|$ is the canonical norm on $\boldsymbol{Q}$. We define a map $\phi: S^{3}(1) \rightarrow S^{2}(1 / 2)$ by $\phi(a)=(1 / 2) \bar{a} \cdot i \cdot a(\bar{a}$ is the conjugate to $a \in \boldsymbol{Q})$. This is well-defined. Then $\psi: S^{3}(1) \rightarrow S^{2}(1 / 2)$ is the hopf fibering and a riemannian submersion with connected totally geodesic fibres (cf. [2]). Thus $S^{3}(1)$ is a foliated riemannian manifold of codimension two with leaves as fibres and the metric is a bunlde-like metric. The leaf through $a \in S^{3}(1)$ is given by the intersection of the plane in $R^{4}$ spanned by vectors $a$ and $i \cdot a$ with $S^{3}(1)$. The tangent space at $a \in S^{3}(1)$ is spanned by $\imath \cdot a, \jmath \cdot a, k \cdot a$. An orthonormal adapted frame $\left\{X_{1}, X_{2}, X_{3}\right\}$ is given by $X_{1}(a)=\imath \cdot a, X_{2}(a)=\jmath \cdot a$ and $X_{3}(a)=k \cdot a$. Take a great circle (geodesic) $\sigma(t)$ parametrized by arc-length and suppose that, at $t=t_{0},\langle\dot{\sigma}(t)$, $\left.X_{2}\right\rangle\left.\right|_{t=t_{0}}=\left.\left\langle\dot{\sigma}(t), X_{3}\right\rangle\right|_{t=t_{0}}=l\left(t_{0}\right) \neq 0$. Then $\sigma(t)$ is the intersection of the plane in $R^{\prime}$ spanned by vectors $\sigma\left(t_{0}\right)$ and $\dot{\sigma}\left(t_{0}\right)$ with $S^{3}(1)$. Therefore, we have $\left\langle\dot{\boldsymbol{\sigma}}(t), X_{2}\right\rangle$ $=\left\langle\dot{\sigma}(t), X_{3}\right\rangle=l(t)=$ constant $=l\left(t_{0}\right)$ for every $t$.

\section{REFERENCES}

1. BoT 1 R., Lectures on characteristic classes and foliations, Lecture Notes in Math. 279, Springer-Verlag. (1972) 1-91.

2. Escobales, R. H., Jr., Riemannian submersions with totally geodesic fibers, J. Diff. Geom. 10 (1975), 253-276.

3 KitahAra, H., The existence of complete bundle-like metrics, Ann. Sci. Kana. zawa Univ. 9 (1972), 37-40.

4 Kitaliara, H., The existence of complete bundle-like metrics, II, Ann. Sci. Kanazawa Univ. 10 (1973), 51-54.

5 KitAllarA, H. AND S. YOROZU, On the cohomology groups of a manifold with a nonintegrable subbundle, Proc. Amer. Math. Soc. 59 (1976), 201-204.

6. Muro, Y., On some properties of a fibred Riemannian manifold, Sci. Rep. Yokohama Nat. Unıv. Sect I. 1 (1952), 1-14.

7 Naveira, A. M., Variedades foliadas con metrica casifibrada, Collect. Math. $21(1970), 41-97$.

8 PASTERVACK, J., Topological obstructions to integrability and the Riemannian geometry of smooth foliations, Thesis, Princeton Unıv., 1970.

9 P.Aster\ACK, J., Foliations and compact Lie group actions, Comment. Math. Helv. 46 (1971), 467-477.

$10^{-}$ReINIART, B., Foliated manifolds with bundle-like metrics, Ann. of Math. 69 (1959), 119-132.

11. SACksteder, R., Foliations and pseudogroups, Amer. J. Math. 87 (1965), 79-102.

12] VAISMAN, I., Variétés riemanniennes feuilletées, Czechoslovak Math. J. 21 (1971), 46-75.

13. Vaismax, I., Cohomology and differentiable forms, Dekker, New York, 1973.

14. Wolf, J.A., Spaces of constant curvature, McGraw-Hill, New York, 1967.

Department of Mathematics

College of liberal Arts

KANAZAWA UNIVERSITY 